\title{
Hyperparathyroidism with presumed sellar-parasellar brown tumour based on imaging findings
}

\author{
Q Said-Hartley, MB ChB \\ S Candy, MB ChB, FCRad (D) \\ Department of Radiology, Groote Schuur Hospital, Cape Town
}

Brown tumours are an end-stage complication of hyperparathyroidism. They are relatively rare today, due to earlier diagnosis and prompt treatment of hyperparathyroidism. ${ }^{1}$ Common locations are the mandible, pelvis, ribs and long bones of the axial skeleton. ${ }^{2}$ The skull base is an extremely rare site and, for obvious reasons, it is difficult to confirm the diagnosis in the absence of other lesions. In the face of a solitary lesion, a histological diagnosis of a 'giant-cell tumour' of benign nature should only be made once hyperparathyroidism has been actively excluded. Brown tumours, solid aneurysmal bone cysts, giant-cell reparative granuloma and giant cell tumour can all appear identical both macroand microscopically. ${ }^{2}$

\section{Case report}

The middle-aged female patient had presented initially 2 years before with a pathological fracture of her left proximal humerus. Radiographs revealed a large lytic and expansile lesion of the left humeral diaphysis, complicated by a pathological fracture. Histology reported a 'giant cell tumour' after surgical resection. A prosthesis was inserted, with no record of further follow-up.

One year later she re-presented with pain in her left shoulder, hip and anterior tibia, and X-rays and bone scans suggestive of 'metastases'. A raised serum calcium was documented, but without any further investigation. She then received radiotherapy, presumably for pain palliation.

After several follow-up visits, a persistently elevated serum calcium (3.51 mmol/l) caused concern and she was admitted for further investigations. These revealed an elevated parathyroid hormone level of 156 pmol/l, with raised alkaline phosphatase (457 U/l) and lacate dehydrogenase $(489 \mathrm{U} / \mathrm{l})$. A repeat bone scan showed the classic appearance of a 'bearded' superscan, due to mandibular involvement (Fig. 1). A sestamibi scan revealed a lesion in the inferior left parathyroid. Numerous other hotspots in the left tibia, pelvis and skull consistent with brown tumours were noted.

Prior to her most recent admission, she presented with headaches and cranial nerve 3, 4 and 5 palsies. CT demonstrated a destructive base of skull lesion (Fig. 2), as well as the maxillary and mandibular lesions shown on the bone scan. MRI T1-weighted post-gadolinium sequences confirmed that this enhancing base of skull lesion invaded the right cavernous sinus and optic canal (Figs 3 - 5).

Based on the above findings, a presumptive diagnosis of a brown tumour of the skull base was made.

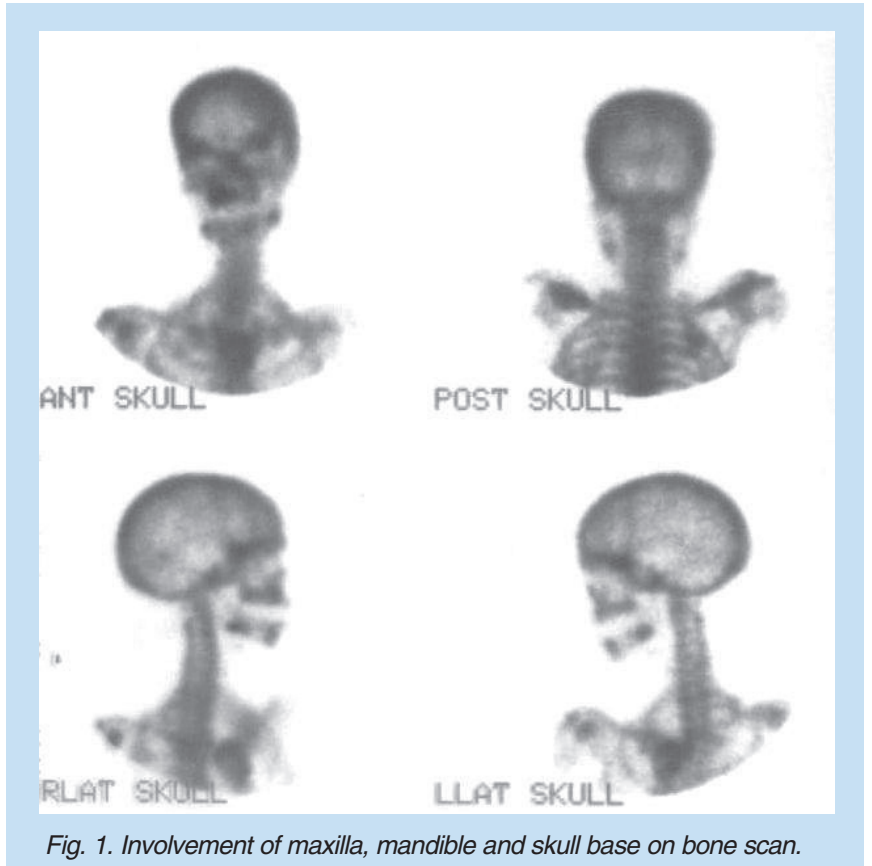

Fig. 1. Involvement of maxilla, mandible and skull base on bone scan.

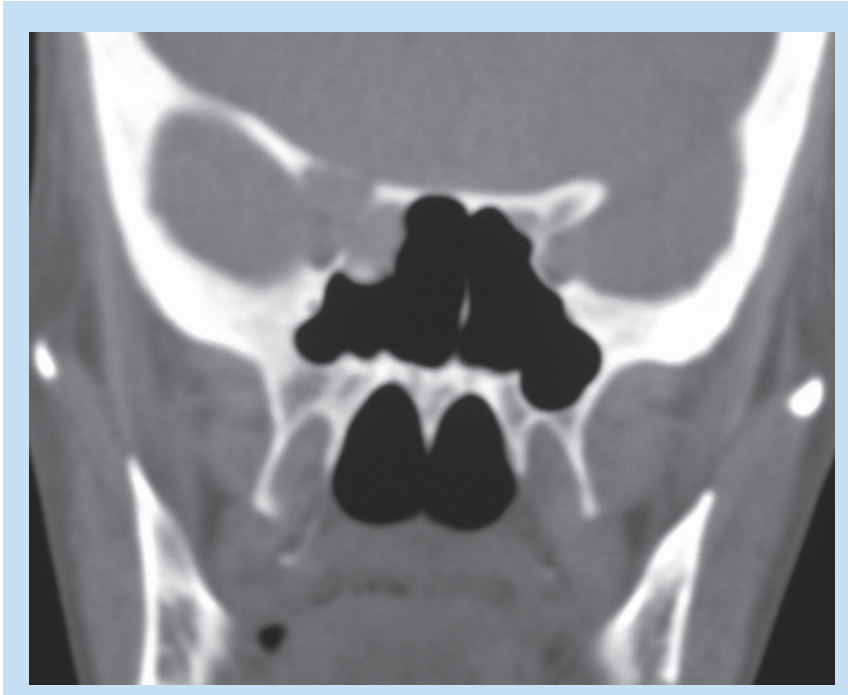

Fig. 2. CT shows expansile, lytic lesion of the right skull base and anterior clinoid process.

\section{Discussion}

As mentioned, brown tumour of the base of skull is exceedingly rare, with only four cases with sellar involvement reported in the English literature on hyperparathyroidism, and two with optic nerve involvement. ${ }^{3,4}$ The differential diagnosis of an expansile, erosive sellar-para- 


\section{CASE REPORT}

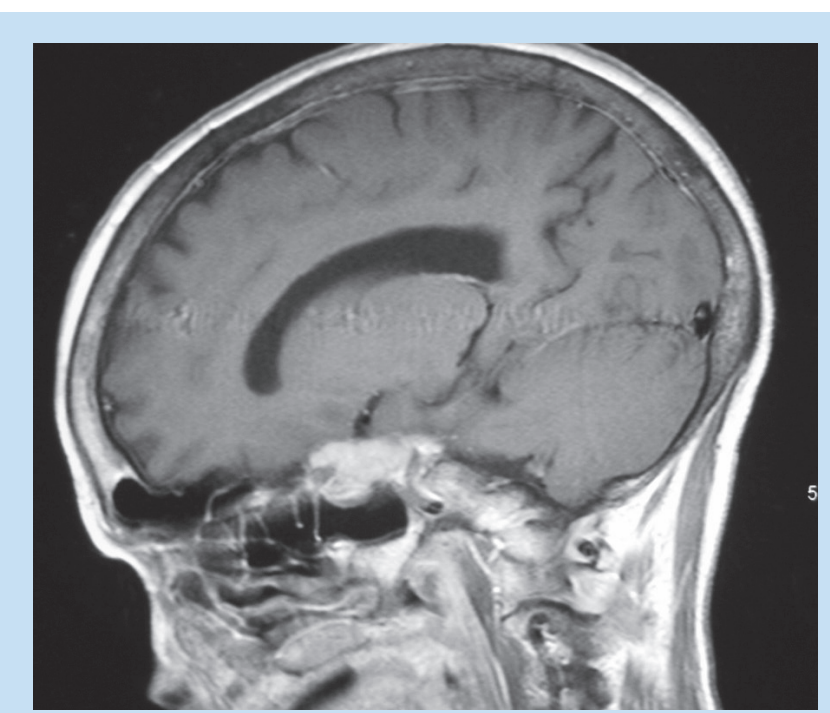

Fig. 3. T1W gadolinium-enhanced MRI shows enhancing right parasellar mass.

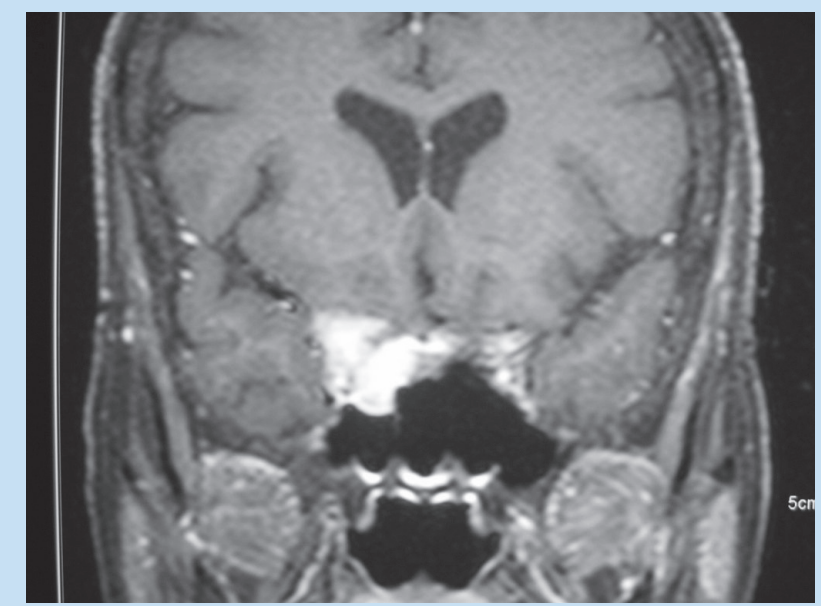

Fig. 4. Enhancing right parasellar/paracavernous mass on coronal postgadolinium imaging.

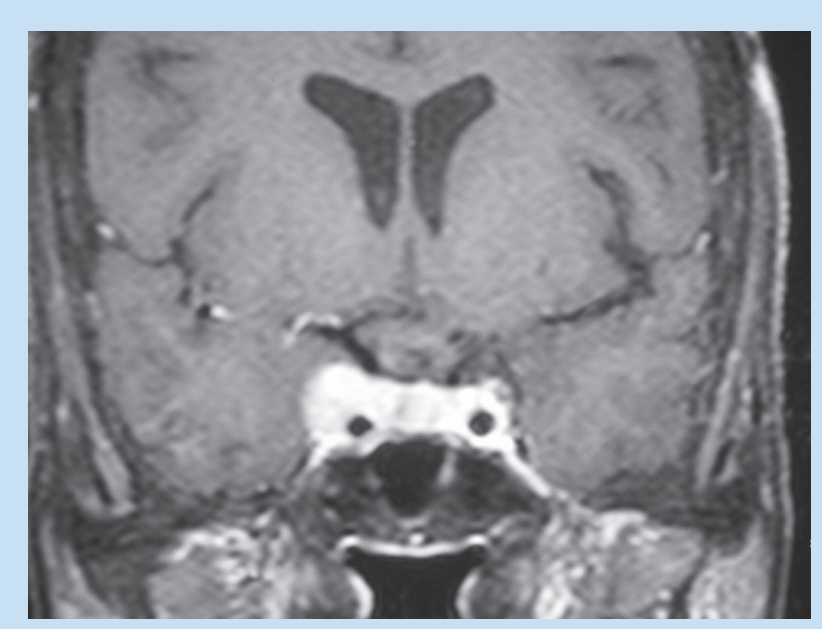

Fig. 5. Extension into right cavernous sinus.

sellar mass with optic nerve involvement includes pituitary pathology, metastatic carcinoma, sarcoidosis, fibrous dysplasia, giant-cell reparative granuloma, chordoma, chondrosarcoma, plasma cell tumour and osteosarcoma. Hyperparathyroidism should be included when subperiosteal bone resorption is present.

In view of the histological appearance and similarity to other giant-cell tumours and similar lesions, brown tumour must always be considered in the differential diagnosis and active steps be undertaken to exclude hyperparathyroidism.

1. Goswami P, Sarma PK, Sethi S, Hazarika S. Skeletal manifestations in a case of primary hyperparathyroid -ism caused by parathyroid adenoma. Ind J Radiol Imag 2002; 12: 267-270.

2. Bone Tumours: Clinical, Radiologic and Pathologic Correlations. Philadelphia: Lea and Febiger, 2006

3. Magu S, Mathur SK, Gulati SP, Yadav A, Kaushal V. Giant cell reparative granuloma of the base of the skull presenting as a parapharyngeal mass. Neurol India 2003; 51: 260-262.

4. Yilmazlar S, Arslan E, Aksoy K, Tolunay S. Sellar-parasellar brown tumor: case report and review of litera ture. Skull Base 2004; 14: 163-168. 